\title{
Introduction to the Special Issue on Social Isolation Across the Lifespan
}

\author{
Suzanne Brown ${ }^{1} \cdot$ Michelle R. Munson $^{2}$
}

Published online: 11 February 2020

(c) Springer Science+Business Media, LLC, part of Springer Nature 2020

Social isolation, having few social network ties and infrequent social contact, is associated with early mortality and poor health outcomes such as cardiovascular disease (Grant et al. 2009), poorer immune system functioning (Cohen 2001), and health behaviors (Kobayashi and Steptoe 2018). Loneliness, the subjective emotional state often (although not always) associated with social isolation also has deleterious effects on mortality risk (Perissinotto et al. 2012), health outcomes (Cacioppo et al. 2002), and general well-being (Booth 2000). While social science research has increasingly studied social isolation as a predictor of poor outcomes, the popular literature has brought the public's attention to both the negative effects of isolation and the socio-economic facilitators of increased isolation over the past 20-years in the United States. For example, Johan Harri's "Lost Connections" published in 2018, took on the subject of depression in the U.S. and examined the associations between commonly considered causes of depression and social isolation as part of the causal mechanism leading to depression; while Robert Putnam's "Bowling Alone" (2000) addressed the socioeconomic and cultural drivers and consequences of social isolation in the United States.

In spite of the increased attention given to social isolation in research and clinical practice over the past 20 years, gaps remain in our understanding of the phenomena of social isolation and loneliness, and in our ability as social workers to effectively intervene. Often used interchangeably, social isolation and loneliness are distinct and separate constructs. Yet, we know little about the ways in which specific interventions affect either one or both of these constructs. The impact and consequences of social isolation have rarely been

Suzanne Brown

suzanne.brown@wayne.edu

1 School of Social Work, Wayne State University, Detroit, MI, USA

2 Silver School of Social Work, New York University, New York, USA considered within a developmental framework. For example, we know little about the impact of childhood isolation on developmental processes across the life course, or interventions that minimize the potential for isolation across the life course. Additionally, research and practice have paid little attention to the interaction between social isolation and social marginalization or the context-specific and differential experiences of isolation and loneliness within specific populations. In this special issue we call for a more nuanced, developmental, complex, and contextualized understanding of social isolation and loneliness.

In 2015 Social Work initiated a campaign, the Grand Challenges for Social Work, to engage practitioners, researchers and policy advocates to work toward eradicating some of society's most pressing concerns. Eradicating Social Isolation is one such grand challenge.

In keeping with this grand challenge, the purpose of this special issue is to move our understanding of the causes and consequences of social isolation and loneliness forward, and to embed our understanding of social isolation and loneliness within developmental and social justice frameworks. The articles in this special issue examine social isolation and loneliness from many different perspectives, within many different populations, across the developmental life span. These articles challenge us to consider the consequences of social isolation and loneliness within distinct developmental periods-childhood, adolescence, young, middle, and older adulthood, for individuals, and within family systems. These articles also remind us of the importance of Social Work's person-in-environment perspective in assessment and intervention as they present research and interventions related to marginalized populations, such as violence exposed AfricanAmerican adolescents, women with HIV, LGBTQ+ elders, and Latinx immigrants. 


\section{Children, Youth, Families, and Young Adults}

The first article in this special issue addresses the importance of youth mentors in the lives of children with supportive non parental adults, and the role of parents as gate-keepers to these relationships. Weiler, Scafe, Spencer, and Cavell, in their article "Caregiver-initiated mentoring: Developing a working model to mitigate social isolation", explore the possibilities and potential for an approach that supports parents' capacity to be gatekeepers to the support networks of their children. Semi-structured interviews were used to interview parents and professionals attending a collaborative workshop to generate strategies to support parents in helping their children cultivate supportive relationships with non-parental adults in their lives. Findings from the study led to the development of a CaregiverInitiated Mentoring approach that can be used by clinical social workers and other helping professionals to assist youth in developing healthy relationships and to increase their network of supportive relationships.

In early development children are embedded within family systems, and their health dependent on the health and supports of their caregivers. Family systems theorists have long elucidated the ways in which the health and well-being of one family member generates effects across family members within the system. In "The association between social isolation and health: An analysis of parent-adolescent dyads from the Family Life, Activity, Sun, Health, and Eating Study", Thompson, Rodebaugh, Bessaha, and Sabbath identify the relationship between parent social isolation and the health of their adolescent children. Using data from a sample of 1851 families they found links between parent social isolation and parent health, adolescent isolation and adolescent health, and parent isolation and adolescent health. Their findings highlight the reciprocal relations between family members in health and isolation and underscore the importance for social workers to consider family-based interventions to address the consequences of parental and child isolation.

In "That's why I stay to myself: Marginalized youth's meaning making processes of social disconnectedness" the authors identify the protective aspects of isolation as expressed by youth exposed to adversity. Storer, McLeary, Pepin, and Stallings conducted six focus groups in the urban South with primarily African American youth aged 16-24. Using thematic analysis as their data analytic strategy their findings highlight the theme of "staying to myself" and the ways in which youth constructed social isolation as a protective mechanism by which they maintained their safety amidst the threat of community and interpersonal violence. These findings remind us that social isolation is a complex construct with idiosyncratic and context-dependent meaning. Consistent with the Strengths perspective and the person-in-environment perspective of Social Work, these findings illustrate that behaviors that appear on the surface to be dysfunctional can be both functional and resilient within their engendering context.

In "Reducing social isolation through formal youth mentoring: Opportunities and potential pitfalls", Keller, Perry, and Spencer discuss how intergenerational relationships created through formal youth mentoring programs have the potential to reduce social isolation among youth, while also offering the opportunity to strengthen community connections among individuals from different backgrounds. They discuss how a relationship-based mentoring approach, if done well, could offer a solution for the increasing isolation among youth in our current society. The authors also explicate that ethical issues and issues around power and social justice need to be addressed directly so as to not reproduce negative outcomes.

In "Eating disorders in 'Millennials': Risk factors and treatment strategies in the digital age", Lenza explores the common risk factors, including social media and "apps," that precipitate eating disorder symptoms among young adults. Lenza then provides a new perspective on treatment for young adults with an adapted Acceptance and Commitment Therapy approach. The approach provides one clinical strategy to address loss of connection and isolation due to social media use with the aim to reduce behavioral health disorders, such as eating disorders and depression so often associated with social isolation.

\section{Middle Adulthood and Social Marginalization}

For social workers working with individuals who experience psychotic symptoms, patterns of social isolation are a common target for intervention. Isolation, caused by psychotic symptoms such as fear, amotivation, and hallucinations may also place individuals at risk for suicide and suicidal ideation. According to authors Bornheimer, Li, Im, Taylor, and Himle, suicide is a leading cause of death among individuals with psychotic symptoms. In their article, "The role of social isolation in the relationships between psychosis and suicidal ideation" these authors found that social isolation does in fact mediate the relationship between psychotic symptoms and suicidal ideation. In other words, the presence of psychotic symptoms increases social isolation, and increased isolation places one at greater risk for suicidal ideation. These findings are a call for practitioners to consider and advocate for treatment beyond the much-advertised psychopharmacological interventions for individuals with psychotic 
disorders, and establish the centrality of relationship for those with serious and persistent mental health disorders.

Stigma refers to an attribute that discredits one socially and marks them as undesirable within their community. Goffman (1963) theorized stigma as the relationship between the discredited attribute, one's own experience of the attribute, and the interpersonal response to the attribute as conditions that may facilitate social isolation or protect against it. In "They kept away: Social isolation of cisgender women living with HIV in Hyderabad, India" authors Azhar, Gandham, Vaudrey, Oruganti, and Samuel utilized thematic analysis to interpret findings from qualitative interviews with sixteen cisgender women living with HIV in Hyderabad. Major content themes illustrate the women's struggles with social isolation as a result of HIV stigma and identify pockets of resilience and hope in the midst of this. The authors end with a discussion of the importance of culturally embedded interventions for this population, and risk for secondary traumatic stress for clinicians working with such stigmatized and isolated groups.

In "Evaluating and intervening in the trauma of solitary confinement: A Social Work perspective", the author both challenges social work to address the negative consequences of solitary confinement-an extreme form of social isolation-and offers a framework for intervening to reduce the negative effects this violent intervention causes human beings. Pforte lays out the Conservation of Resources (COR) theory side-by-side with a discussion of structural racism in society. Pforte then discusses three promising interventions for addressing the trauma prisoners experience when confined in a cell, namely (1) nature imagery, (2) Clinical Alternative to Punitive Segregation (CAPS), and (3) the Sanctuary Model, and illustrate where these interventions align with COR theory. He concludes with recommendations for social work practice, policy and research.

Previous research and theorizing on social isolation and social support have not explicated the specific effects of objective isolation (quantifiable social connections) versus subjective isolation (perceived level of closeness) on physical or mental health outcomes. Additionally, research examining the impact of social isolation specifically within racial and ethnic minority populations is scant. Authors Nguyen, Taylor, Taylor, and Chatters aim to address these gaps in their paper "Objective and subjective social isolation and psychiatric disorders among African Americans". The authors examined the impact of objective and subjective isolation on major depressive disorder, presence of any Diagnostic and Statistical Manual of Mental Disorders (DSM) diagnosis, and number of DSM diagnoses 12 months following measures of isolation. They found that subjective social isolation from both family and friends was associated with the presence of major depression, the presence of any DSM diagnosis, and number of diagnoses; whereas objective isolation had no effect on likelihood of having depression or any psychiatric diagnosis. This study furthers our understanding of within group experiences of isolation and its impact on mental health for African Americans.

Latinx immigrants are the largest immigrant group in the United States. Immigration is frequently stressful, made more so for Latinx individuals by the current political climate and increasing risk for potential isolation. Resilience, the capacity to recover from difficulties and adverse experiences, is a vital protective factor in the face of life stressors, transitions and disruptions such as immigration. Lee, Hong, Zhou, and Robles, in their article entitled "The relationships between loneliness, social support, and resilience among Latinx immigrants" centered resilience among Latinx immigrants in their investigation, with the aim of identifying the impact of social support and loneliness on resilience. Greater social support and lower loneliness were associated with greater resilience in this population. The authors stressed the importance of comprehensive interventions for this population, that span micro, mezzo, and macro levels, cognitive interventions, social support interventions, and the early settlement houses. The context dependent nature of concepts like loneliness and isolation are highlighted by these authors and the ways in which marginalized identity and status interact with other social and behavioral factors to intensify loneliness and isolation.

Learning more about the causes and consequences of social isolation across the developmental life course and within specific populations then begs the question, "what do we do about it?". One starting place may be "A systematic review of loneliness interventions among non-elderly adults" by authors Bessaha, Sabbath, Morris, Malik, Scheinfeld, and Saragossi. In this paper the authors reviewed 68 studies of interventions designed to reduce social isolation among those with mental health issues, individuals with disabilities, those with chronic medical conditions, members of the military, parents and caregivers, and immigrants and refugees. They identified a significant reduction in loneliness for participants who engaged in technology-based interventions and support group interventions. The authors synthesized information from their review into specific recommendations for intervention, including the use of peer led interventions to reduce loneliness especially within marginalized populations.

\section{Older Adulthood}

LGBTQ+ older adults are at high risk for social isolation, as they are significantly more likely to live alone and to have no children than their heterosexual counterparts. Additionally, issues of stigma, particularly for cohorts of aging LGBTQ+ individuals who came of age well before 
same sex marriage and LGBTQ+ civil rights legislation, the need to hide aspects of self in order to maintain safety, with concomitant social isolation, may have been a lifelong experience. Perone, Ingersoll-Dayton, and WatkinsDukhie address marginalization and social isolation among LGBTQ+ older adults in their paper, "Social isolation and loneliness among LGBTQ+ older adults: Lessons learned from a pilot Friendly Caller Program". Using Community Based Participatory Action methods, the authors identified, implemented and evaluated a volunteer program that trained volunteers to be "friendly callers" to provide support for socially isolated LGBT elders. While these intergenerational matches helped to decrease isolation among the elders the project also identified those at risk for isolation among the volunteer group themselves and in some cases decreased isolation among both those targeted for intervention and those offering support.

The final article in this special issue further develops our understanding of the conceptual complexity of social isolation as both construct and experience. In "Social isolation's influence on loneliness among older adults", Taylor operationalizes social isolation into seven component parts including isolation from adult children, from other family members, from friends, living alone, being unmarried, not participating in social groups, and isolation from religious activities. Taylor addresses the question of which isolation indicators are most influential in determining loneliness specifically among older adults. His findings underscored the importance of isolation from family and friends, and lack of participation in social groups or religious activities as significant risk factors for greater loneliness. Social isolation is multi-faceted making it difficult to target interventions toward the most influential aspects of isolation. This article furthers our understanding of the role of specific aspects of isolation, offering a potentially more nuanced approach to interventions.

\section{Conclusion}

Each article in this special issue demonstrates that individuals and families struggle with social isolation and loneliness across the developmental spectrum and speaks to the importance of Social Work's Grand Challenge to Eradicate Social Isolation. Isolation and loneliness manifest in different ways for different people, suggesting the importance of clinicians assessing both the objective qualities of clients' social networks as well as their felt sense of loneliness. Interventions that cross the micro, mezzo, and macro levels are important to combatting social isolation, especially as structural barriers related to stigma and marginalization intensify isolation. The use of technology, while in some cases emblematic of social isolation, may in other cases be a useful platform for interventions that reduce isolation. Peer support and the facilitation of peer helping relationships, especially for those with stigmatized identities, may be both empowering and decrease isolation for populations such as LGBTQ+ elders, individuals with HIV, and immigrants. Family based interventions to decrease loneliness and isolation among parents can have ripple effects on the health and well being of children, as can interventions that enable parents to facilitate mentoring relationships for their children.

As co-editors of this Special Issue, our goal was to bring together a diverse set of conceptual and empirical papers that begin to elucidate the complex interplay between developmental period, social location, and social isolation. Methodologically these papers utilize a range of quantitative research methods that identify causes and consequences of isolation and loneliness, as well as qualitative methods that give voice to those for whom experiences of stigma and social exclusion are inextricably linked to social isolation. It is our hope that this special issue will initiate a sustained interest in working across micro, mezzo, and macro levels to reduce social isolation in a society where isolation and loneliness are increasingly problematic. As a profession, Social Work is uniquely positioned to approach human suffering from both developmental and social justice perspectives. We hope that this special issue will allow for the conceptual framing of social isolation as a phenomenon with implications across the life course, and that it moves you, the reader, to a deeper, more nuanced, and contextualized understanding of social isolation and loneliness.

\section{References}

Booth, R. (2000). Loneliness as a component of psychiatric disorders. Medscape General Medicine, 2, 1-7.

Cacioppo, J. T., Hawkley, L. C., Crawford, L. E., Ernst, J. M., Burleson, M. H., Kowalewski, R. B., et al. (2002). Loneliness and health: Potential mechanisms. Psychosomatic Medicine, 64, 407-417.

Cohen, S. (2001). Social relationships and susceptibility to the common cold. In C. D. Ryff \& B. S. Singer (Eds.), Emotion, social relationships and health (pp. 221-232). New York: Oxford University Press.

Goffman, E. (1963). Stigma, notes on the management of spoiled identity. New York: Simon and Schuster.

Grant, N., Hammer, M., \& Steptoe, A. (2009). Social isolation and stress-related cardiovascular, lipid, and cortisol responses. Annals of Behavioral Medicine, 37, 29-37. https://doi.org/10.1007/s1216 0-009-9081-z.

Harri, J. (2018). Lost connections. New York: Bloomsbury Publishing.

Kobayashi, L. C., \& Steptoe, A. (2018). Social isolation, loneliness, and health behaviors at older ages: Longitudinal cohort study. Annals of Behavioral Medicine, 52(7), 582-593. https://doi. org/10.1093/abm/kax033.

Perissinotto, C. M., Stijacic Cenzer, I., \& Covinsky, K. E. (2012). Loneliness in older persons: A predictor of functional decline and death. Archives of Internal Medicine, 172, 1078-1083. https ://doi.org/10.1001/archinternmed.2012.1993.

Putnam, R. (2000). Bowling alone. New York: Simon and Schuster. 
Publisher's Note Springer Nature remains neutral with regard to jurisdictional claims in published maps and institutional affiliations.

Suzanne Brown is Associate Professor of Social Work at Wayne State University's School of Social Work in Detroit, Michigan. Her research and scholarship focus on the intersection of trauma and substance use disorders among women; parenting processes among women with histories of child maltreatment, violence exposure, and substance use disorders; early bonding and social support as protective factors for women with substance use disorders.

Michelle R. Munson is Professor of Social Work at New York University's Silver School of Social Work in New York city. Her research and scholarship are in the areas of mental health services and interventions; mood, anxiety, trauma and stress-related disorders; influence of social relationships; social isolation; service use decisions; and the developmental transition from adolescence to adulthood. 